\title{
Investigation on Deformation of the Flex-Gear and Optimization Design of Compound Curve Cam Wave Generator with Spiral
}

\author{
Shuyan Wang $\mathbb{D},{ }^{1}$ Yudelong Zhang, ${ }^{1}$ Shiteng Mao $\mathbb{D},{ }^{1}$ and Yu He $\mathbb{D}^{2}$ \\ ${ }^{1}$ College of Mechanical Engineering, Donghua University, Shanghai 200051, China \\ ${ }^{2}$ School of Urban Rail Transportation, Shanghai University of Engineering Science, Shanghai 201620, China
}

Correspondence should be addressed to Shuyan Wang; shuyan@dhu.edu.cn

Received 3 September 2021; Accepted 14 September 2021; Published 25 September 2021

Academic Editor: Debiao Meng

Copyright ( 2021 Shuyan Wang et al. This is an open access article distributed under the Creative Commons Attribution License, which permits unrestricted use, distribution, and reproduction in any medium, provided the original work is properly cited.

The deformation shape and mechanical property of flex-gear greatly affect the meshing accuracy and service life of harmonic drive, and the stress wave generator plays a decisive role in the deformation shape and mechanical properties of the flexible gear. The investigation on deformation of the flex-gear around the major axis is developed with comparative analysis with three kinds of traditional and commonly used wave generators. Based on the influence of the deformation around the major axis on meshing accuracy and service life, the eccentric arc cam wave generator improved by transition curve for controlling the deformation shape and reducing the deformation stress is developed. Considering the achievable flexibility of spiral with smooth curvature change rate, the contour of the compound curve cam wave generator with arc and spiral is proposed. The deformation of the flex-gear under the influence of this compound curve cam and the standard elliptic cam is further comparative analyzed. The conclusion shows that this compound curve cam can indeed reduce the deformation stress in the meshing area by increasing the meshing width, and correct deformation shape can also be gained by shape control on the whole circumference of the flex-gear's neutral layer.

\section{Introduction}

In harmonic drive, the flex-gear is in the severe condition of infinite alternating asymmetric cyclic stress, and the continuous deformation which affects the meshing quality and service life mainly depends on the deformation caused by the stress wave generator. Rather than the conventional single curve, the cam stress wave generator designed with the compound curve can effectively improve the mechanical deformation in the meshing area of the flexible gear. In the compound curve design, the types of curves, the parameters of curves, and even the curvature change rate of curves would all affect the mechanical property of the flex-gear $[1,2]$, so curve design and optimization [3-6] of the compound curve cam wave generator for lower deformation and high meshing quality is a main research domain in harmonic drive.

In order to improve the meshing performance and extend the service life of harmonic gear transmission, scholars have made a lot of efforts in this regard. Yague [7] analyzed the von Mises stress generated by four different wave generators on the flex-gear. Gravagno [8] analyzed the kinematic errors caused by different wave generator profiles and derived the parametric equation between flex-gear and fixed circular spline. Bhabani [9] used the finite element method and experiments to compare the strain and stress of the flex-gear acted by the traditional and split cam generator. $\mathrm{Li}$ [10] conducted static and transient dynamic analysis of flex-gear under the action of typical contours. Routh [11] obtained the hydrodynamic lubrication equation of the interface between the flex-gear and wave generator by solving the generalized Reynolds equation. Vladis [12] discussed the angular displacement between the meshing area and the wave generator and established system equations among flex-gear, circular spline, and wave generators based on contact deformation. Fan [13] obtained the deformation relationship based on the plate and shell theory and further derived the neutral layer deformation equation. 
Han [14] studied the stress and related vibration characteristics of the flex-gear with the numerical analysis tool. $\mathrm{Hu}$ [15] analyzed the influence of some geometric structural parameters such as the aspect ratio of the cup, the wall thickness, the round corner radius, and the width of the tooth ring on internal stress of the flex-gear. Yang [16] studied the influence of the flex-gear geometric parameters on the stress value and distribution for the short cup flexgear. Ostapski $[17,18]$ proposed a method to solve the elastic deformation problem of the thin-walled shell structure with complex shape in the geometrically nonlinear shell theory. Sahoo [19] proposed a method to calculate contact tooth load and flex-gear stress by estimating the contact load of a large number of teeth pairs. Yang [20] proposed an accurate solution for the conjugate tooth profile of the cam wave generator harmonic drive. Xing [21] found that the radial displacement of the minor axis area of the flex-gear is less than the theoretical value, and there is a large film stress in the neutral layer of the flex-gear. Xin [22] simplified the gear tooth profile shape to triangle and trapezoid and compared the effect of the simplified tooth shape on the flex-gear. Zhang [23] analyzed the effect of radial deformation on the meshing performance of harmonic drive and obtained the stress distribution of the flex-gear deformed with the cam wave generator through finite element analysis. Dong [24] studied the deformation of the flex-gear and analyzed the mathematical model of the flex-gear.

In this study, main factors influencing the deformation shape and mechanical properties of the flex-gear are investigated such as the type of the stress wave generator, the curve's saturation level in the working area, and curvature change rate of the wave generator. The design principle of the wave generator for lower deformation and good meshing quality is developed. In the compound curve cam wave generator, spiral curve combined with arc is further proposed with correct parameters, and theoretical design, numerical comparative analysis, and finite element analysis on this compound curve cam wave generator have been carried out.

\section{Conventional Cam Stress Wave Generator with Single Curve}

Among various wave generator types, cam type can make the meshing of the flexible gear and the rigid wheel reach the ideal state with stable operation, high precision, and high efficiency. More importantly, the stress distribution of the flexible gear acted by the cam wave generator could be improved, and the bearing capacity would be higher than other wave generators. Nowadays, cam wave generators mainly include double eccentric arc cam, cosine cam, and standard ellipse. For the convenience of research, the contour equation of cam wave generators is unified as

$$
\left\{\begin{array}{l}
x=x(t) \\
y=y(t)
\end{array}\right.
$$

where $x$ and $y$ are the coordinate points of cam wave generator's profile, and $t$ indicates the angular variable.
(1) When standard ellipse is chosen for the cam wave generator, the parametric equation is given as follows:

$$
\left\{\begin{array}{l}
x=a \cos (t) \\
y=b \sin (t)
\end{array}\right.
$$

Here, $a$ is the semimajor axis of the standard ellipse, and $b$ is the semiminor axis of the standard ellipse.

(2) When cosine curve is chosen for the cam wave generator, the parametric equation is given as follows:

$$
\left\{\begin{array}{l}
x=\left(0.5 d_{b}+\omega_{0} \cos (2 t)\right) \sin (t) \\
y=\left(0.5 d_{b}+\omega_{0} \cos (2 t)\right) \cos (t) .
\end{array}\right.
$$

Here, $d_{b}$ is the basic circle diameter of the cosine curve cam wave generator, and $\omega_{0}$ is the amplitude of the cosine curve.

(3) When double eccentric arc is chosen for the cam wave generator, the parameter equation is

$$
\left\{\begin{array}{l}
x=r \cos (t) ; y=r \sin (t)+e, \\
\left(\frac{\pi}{2}-\beta<t<\frac{\pi}{2}+\beta \text { and } \frac{3 \pi}{2}-\beta<t<\frac{3 \pi}{2}+\beta\right), \\
x=r \cos (\beta), \\
\left(\beta-\frac{\pi}{2} \leq t \leq \frac{\pi}{2}-\beta \text { and } \frac{\pi}{2}+\beta \leq t \leq \frac{3 \pi}{2}-\beta\right),
\end{array}\right.
$$

here, $r$ is the radius of the eccentric arc, and $\beta$ is half of the arc angle.

Based on the size of the flexible gear in models $17-80$ (Table 1), the basic parameters of the three cam wave generators are calculated as given in Table 2. The radial deformation and stress of the flex-gear acted by these three different wave generators are developed in FEM, as shown in Figures 1-3.

From Figure 1, the equivalent stress of the flex-gear around the major axis with the double eccentric arc wave generator is much less than with the other two cam wave generators. But from Figure 2, the radial deformation at the major axis is $0.310 \mathrm{~mm}$ with the double eccentric arc wave generator, $0.288 \mathrm{~mm}$ with the cosine curve cam wave generator, and $0.291 \mathrm{~mm}$ with the standard ellipse cam wave generator. The radial deformation at the major axis with the double eccentric arc wave generator is a lot different from the value given in theory, which is bigger and less accurate than with the standard ellipse or cosine curve cam wave generator. Taken together, although the double eccentric arc wave generator can effectively reduce the equivalent stress of the flex-gear around the major axis, the radial deformation of the flex-gear around the major axis is used to ensure the meshing quality deviates from the theoretical setting value. This is mainly due to the perimeter of the double eccentric 
TABLE 1: The parameters of the flex-gear.

\begin{tabular}{lcc}
\hline Parameters & Symbols & Values \\
\hline Diameter of the neutral layer $(\mathrm{mm})$ & $\mathrm{Dm}$ & 41.98 \\
Wall thickness $(\mathrm{mm})$ & $\mathrm{h}$ & 0.26 \\
Width of tooth ring $(\mathrm{mm})$ & $\mathrm{bt}$ & 8 \\
Width of cup $(\mathrm{mm})$ & $\mathrm{b}$ & 23.5 \\
Thickness of cup bottom $(\mathrm{mm})$ & $\mathrm{hw}$ & 3 \\
Outer diameter of cup bottom $(\mathrm{mm})$ & Do & 80 \\
\hline
\end{tabular}

TABLE 2: Basic parameters.

\begin{tabular}{lcc}
\hline Wave generator type & Parameters based on flexible gear in models $17-80$ \\
\hline Standard ellipse cam & $a=15.418 \mathrm{~mm}$ & $b=14.88 \mathrm{~mm}$ \\
Cosine cam & $r_{b}=15.15 \mathrm{~mm}$ & $\omega_{0}=0.268 \mathrm{~mm}$ \\
Double eccentric arc cam & $r=14.48 \mathrm{~mm}$ & $e=0.938 \mathrm{~mm} \beta=30^{\circ}$ \\
\hline
\end{tabular}

arc wave generator is shorter than that of equidistant curve of the flex-gear neutrosphere, which would lead to lose control of the flex-gear's deformation shape around the minor axis, so the deformation at the major axis will deviate from the control shape set by the wave generator and the radial deformation would inevitably increase.

In addition, there is also a stress plateau phenomenon in the stress curve of the flex-gear. From Figure 1, the stress curve of the flex-gear shows a stress plateau about $50^{\circ}$ around the semimajor axis, and then, the deformation stress reduces rapidly to another stress plateau with a much lower stress value around the semiminor axis. The value and the width of the stress plateau area around the major axis with cosine curve type is a litter bigger than with the conventional ellipse cam. It is worth noting that although the radial deformation of the flex-gear around the major axis with the double-arc wave generator is large, the value of the stress plateau is smaller and the width is bigger than with the other two cam wave generators. The analysis of the stress curve shows the following: (1) the value of the stress plateau around the major axis is mainly determined by the maximum deformation given in theory and the contour's shape of the cam; (2) the width of the stress plateau around the major axis is mainly affected by the contour's shape of the cam, which strongly affected the number of meshing teeth; and (3) the velocity of stress reduction between the two stress plateaus depends on the curvature change rate of the cam's contour.

In summary, designing a compound curve cam wave generator with shape control of full perimeter can realize high meshing accuracy, and an appropriate shape around the major axis can reduce the deformation stress and increase the number of meshing teeth to improve the service life.

\section{Design Method of Combined Cam Wave Generator}

There are two points in designing a good combined cam wave generator: one is to choose an appropriate curve for the deformation shape around the major axis and the other is to fit the transition curve between the appropriate curves with continuous curvature for shape control of full perimeter.
3.1. Transition Curve. Transition curve refers to the curve with the continuous curvature set between straight line and circular curve, circular curve and circular curve in plane with the same turning direction. As shown in Figure 3, there are many types of transition curves, such as cyclotron, cubic parabola, seventh quartic, half wave sine, one wave sine, double helix, and multicenter complex curve. In road design, when the curve radius on the main line is relatively small, transition curve should be added between the circular curve and the straight line to realize the gradual transition of the curvature and reduce impact at the mutation point of the traffic. It can make the lateral force of the vehicle gradually change, which is conducive to the safety and stability of driving, and can meet the visual and psychological requirements of the driver. The commonly used transition curve of highway and ramp is spiral, also known as radial spiral, as shown in Figure 4. The curve is not only beautiful but also consistent with the trajectory of the driver turning the steering wheel from a circular curve to a straight line or from a straight line to a circular curve.

The essential characteristic of spiral is given as follows:

$$
\frac{\mathrm{d} k}{\mathrm{~d} l}= \pm \frac{1}{A^{2}}
$$

where $\mathrm{d} k / \mathrm{d} l$ means that the curvature $k$ changes with the arc length $l$, and \pm indicates that the curvature increases or decreases with the arc length. $A$ is the parameter of the spiral, which is the geometric mean of the radius $R$ of the arc segment and the total length of the spiral Ls. The larger $A$ is, the slower the curvature changes and the slower the curve turns. And in reverse, the smaller $A$ is, the faster the curvature changes and the faster the curve turns.

In equation (5), $A$ cannot represent the increase or decrease of curvature. Thus, a is introduced, the relationship between a and $A$ can be expressed as $|a| A^{2}=1$. Equation (5) can be rewritten as follows:

$$
\frac{\mathrm{d} k}{\mathrm{~d} l}=a,
$$

where $a$ is the rate of curvature changing, and it can be positive or negative. 


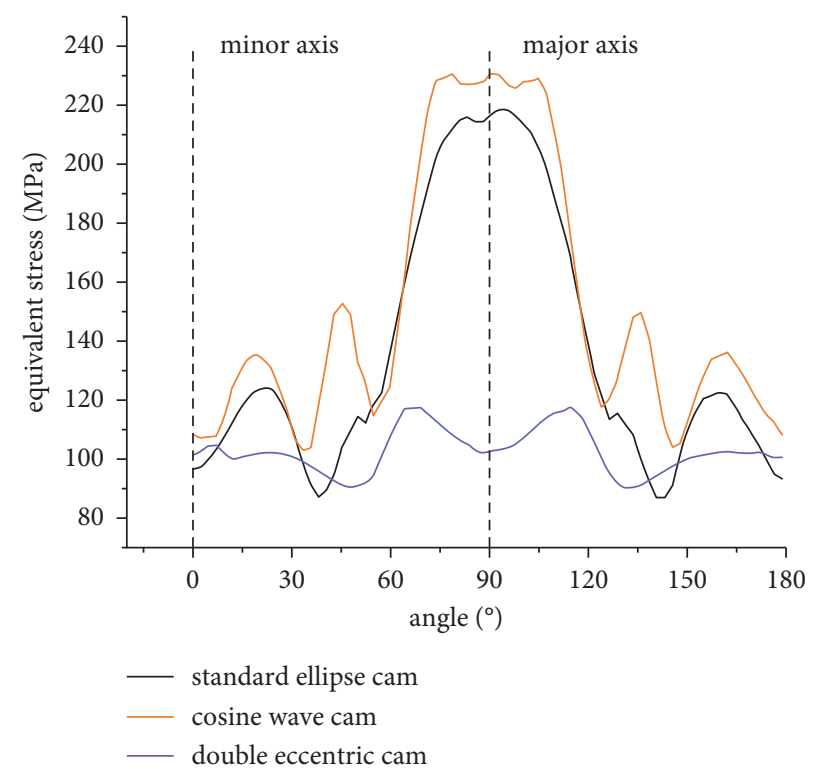

FIGURE 1: Equivalent stress of the flex-gear with three cam wave generators.

In our cam design, considering the disadvantages of the flex-gear's deformation stress with the double-arc wave generator, a compound curve with two arcs and two spiral curves is developed for the contour design of a good combined cam wave generator. Thus, the spiral is smooth and its curvature changes proportionally with the length of the curve, which can maintain the advantages of the double eccentric arc cam wave generator with lower equivalent stress and bigger width of the stress plateau around the major axis and can even more improve the accuracy of the flex-gear's deformed shape.

\subsection{Contour Design of Combined Cam Wave Generator Based} on Spiral. Because the contour of the combined cam wave generator is axisymmetric, only $1 / 4$ of the contour needs to be designed. In order to explain the combination of spiral and arc in the contour of the compound curve cam wave generator, the coordinate systems are setup as shown in Figure 5. The global coordinate system oxy is fixed at the original point of the spiral, the $x$ axis is tangent to the spiral at the original point, and the $y$ axis satisfies the right-hand rule. The local coordinate system $o_{1} x_{1} y_{1}$ is fixed at the center of the arc, and the local coordinate system is parallel to the global coordinate system.

General solutions of spiral's differential equation can be expressed as

$$
\begin{array}{r}
x+i y=x_{0}+i y_{0}+\left(\cos T_{0}+i \sin T_{0}\right) \\
\left\{\left(L-L_{0}\right) \sum_{n=0}^{+\infty} \sum_{r=0}^{n} \frac{i^{n}\left[(a / 2)\left(L-L_{0}\right)^{2}\right]^{r}\left[k_{0}\left(L-L_{0}\right)\right]^{n-r}}{r !(n-r) !(n+r+1)}\right\},
\end{array}
$$

where $x$ and $y$ indicate the coordinate point of spiral, $x_{0}$ and $y_{0}$ indicate the coordinate point of the spiral segment's starting point, $T_{0}$ is the forward direction of the spiral segment's starting point. $L$ is the arc length of the spiral segment's ending point, $L_{0}$ is the arc length of the spiral segment's starting point, and $k_{0}$ is the curvature of the selected spiral segment's starting point.

Because the starting point of the spiral segment is at the coordinate origin of the global coordinate system oxy and the direction of the starting point is tangent to axis $o x$, so $x_{0}=0, y_{0}=0$, and $T_{0}=0$. The differential equation of spiral is simplified as

$$
x+y=l * \sum_{n=0}^{\infty} \sum_{r=0}^{n} \frac{(a / 2 * l)^{r} *\left(k_{0} * l\right)^{n-r}}{r !(n-r) !(n+r+1)}, \quad l \in[0, l s]
$$

where $l$ is the arc length of the spiral segment from the origin point to any point, and $l s$ is the full length of the spiral segment.

In order to control the deformation shape on the whole circumference, the circumference of the compound curve should be equal to that of the neutral layer of the flex-gear. So, the length of the spiral segment can be deduced as

$$
l s=r_{m} * \frac{\pi}{2}-r_{0} * \beta,
$$

where $r_{m}$ is the radius of the flex-gear's neutral layer, $r_{0}$ is the radius of arc segment around the major axis, and $\beta$ is the semienvelop angle of the arc segment.

Because the curvature at the end of the spiral segment is the same as that of the arc segment, the curvature change rate of the spiral segment is

$$
a=\frac{k_{s}-k_{0}}{l s}
$$

where $k_{s}$ is the curvature at the end of the spiral segment and $k_{s}=1 / r_{0}, k_{0}$ is the curvature at the start of the spiral and $k_{0}=1 / r_{r}$, and $r_{r}$ is the curvature radius of the spiral segment's starting point. 


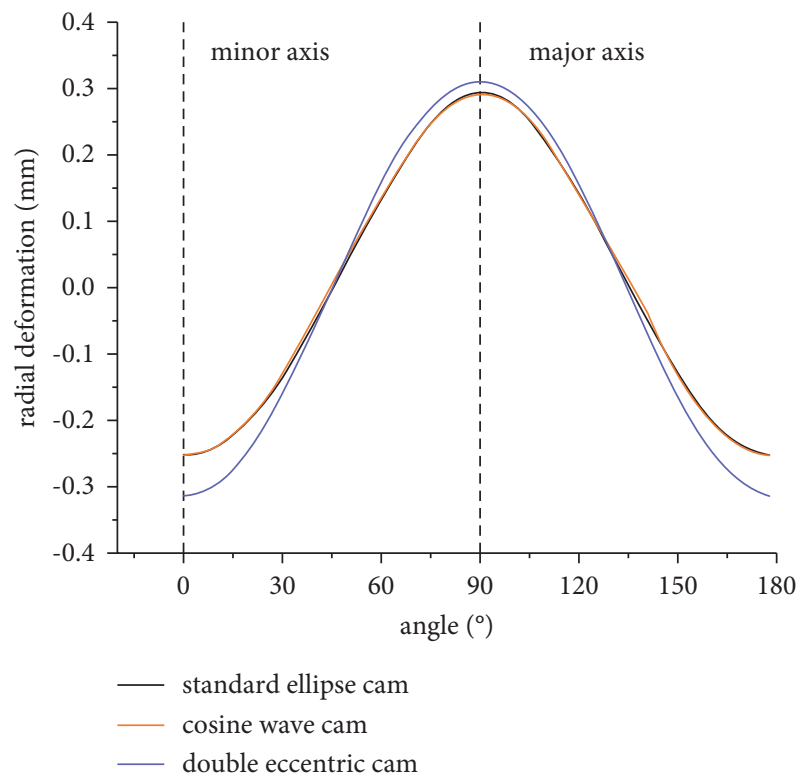

Figure 2: Radial deformation of the flex-gear with three kinds of wave generator.

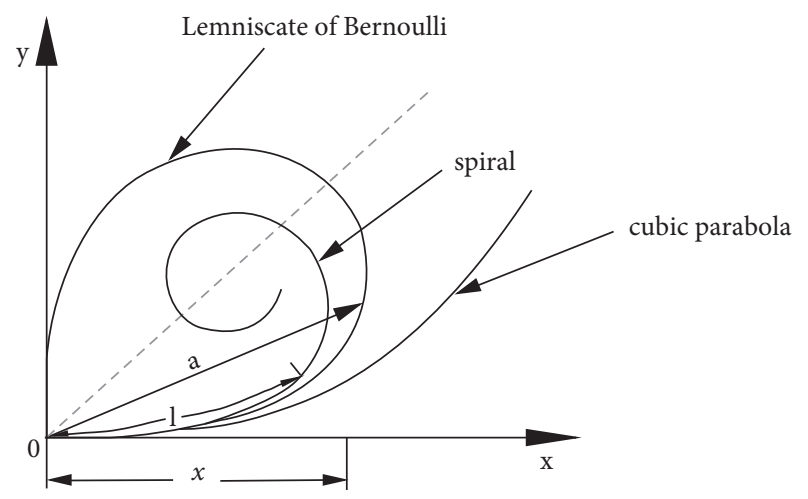

FIgURE 3: Common transition curves.

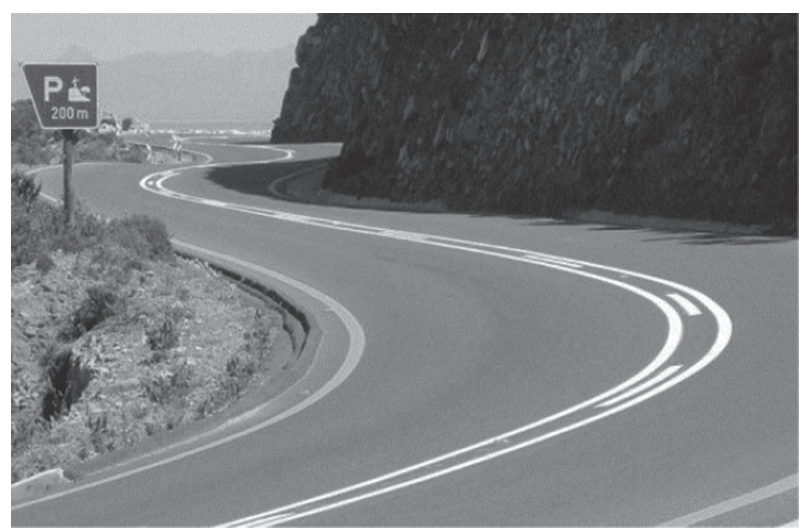

FIgURE 4: Spiral curve in road design.

The coordinates of the end point of the spiral segment in the compound curve cam wave generator can be described as

$$
\left\{\begin{array}{l}
x_{z}=x_{e}+r_{0} * \cos (\beta), \\
y_{z}=y_{e}-r_{0} * \sin (\beta),
\end{array}\right.
$$

where $\left(x_{e}, y_{e}\right)$ is the origin of the local coordinate system $o_{1} x_{1} y_{1}$ and is also the center of the arc segment.

After solving simultaneous equations (8), (9), (10), and (11), the equations of the spiral segment and the arc segments of the flex-gear's center line layer after deformation can be obtained. The equation of the arc segment after deformation can be expressed as

$$
\left\{\begin{array}{l}
x_{r}=r_{0} * \cos (t)+x_{e}, \\
y_{r}=y_{z}+r_{0} * \sin (\beta)+r_{0} * \sin (t) .
\end{array}, \quad t \in[-\beta, \beta] .\right.
$$

Then, the contour curve of the compound curve cam can be obtained by using the equidistant curve, and the equation can be expressed as

$$
\begin{aligned}
& \left\{\begin{array}{l}
x_{w r}=x_{r}+\frac{y_{r}^{\prime}(t) * h}{\sqrt{x_{r}^{\prime}(t)^{2}+y_{r}^{\prime}(t)^{2}}}, \\
y_{w r}=y_{r}-\frac{x_{r}^{\prime}(t) * h}{\sqrt{x_{r}^{\prime}(t)^{2}+y_{r}^{\prime}(t)^{2}}}, \quad t \in[-\beta, \beta],
\end{array}\right. \\
& \left\{\begin{array}{l}
x_{w s}=x+\frac{y_{s}^{\prime}(l) * h}{\sqrt{x_{s}^{\prime}(l)^{2}+y_{s}^{\prime}(l)^{2}}}, \\
y_{w s}=y-\frac{x_{s}^{\prime}(l) * h}{\sqrt{x_{s}^{\prime}(l)^{2}+y_{s}^{\prime}(l)^{2}}}, \quad l \in[0, l s],
\end{array}\right.
\end{aligned}
$$

where $\left(x_{w r}, y_{w r}\right)$ indicates the coordinate point of the arc segment in the compound curve cam, $\left(x_{w s}, y_{w s}\right)$ indicates the coordinate point of the spiral segment, and $h$ indicates the equidistant distance.

\section{Finite Element Analysis and Discussion of the Case Study}

In this compound curve cam wave generator, the radius of the arc segment and the eccentric distance are used as variables for optimization of the deformation shape around the major axis (Table 3), and the radial deformation and the equivalent stress of the flex-gear acted by the compound curve cam with different parameters are developed in FEM, as shown in Figures 6 and 7.

From Figure 6, under the influence of this compound curve cam with arc and spiral, radial deformation of the flexgear at the major axis varied from $0.291 \mathrm{~mm}$ to $0.292 \mathrm{~mm}$ with the value of the eccentric distance $e$ varied from $0.95 \mathrm{~mm}$ to $0.7 \mathrm{~mm}$. Compared with under the influence of double eccentric arc cam, the radial deformation of the flexgear is controlled to a more accurate value from $0.310 \mathrm{~mm}$ to $0.291 \mathrm{~mm}$ or $0.292 \mathrm{~mm}$, which is also less than $0.5 \%$ different from with the conventional ellipse cam. This shows that the 


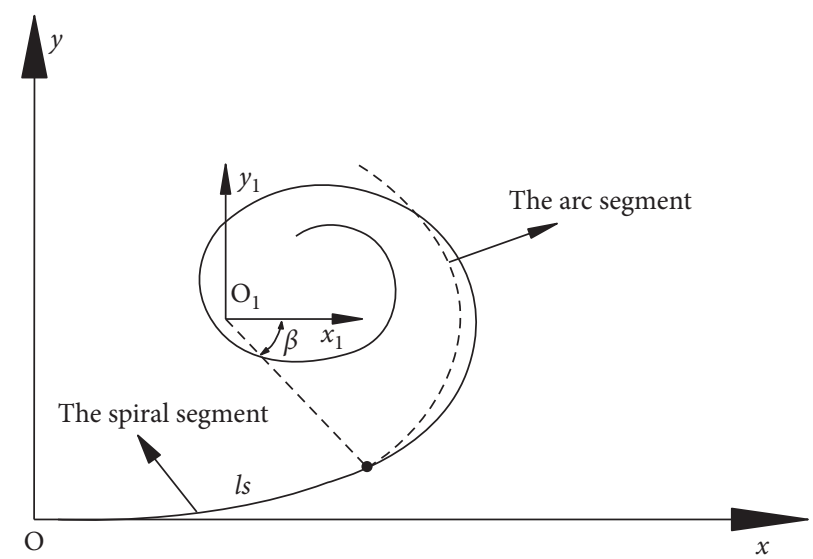

FIgURE 5: Coordinate system of the compound curve.

TABLE 3: The parameters of the wave generator.

\begin{tabular}{lcc}
\hline & Symbols & Values \\
\hline Radius of the arc segment $(\mathrm{mm})$ & $r_{0}$ & $20.558-20.308$ \\
Eccentric distance $(\mathrm{mm})$ & $E$ & $0.7-0.95$ \\
The wrap angle $\left(^{\circ}\right)$ & $\beta$ & $30^{\circ}$ \\
\hline
\end{tabular}

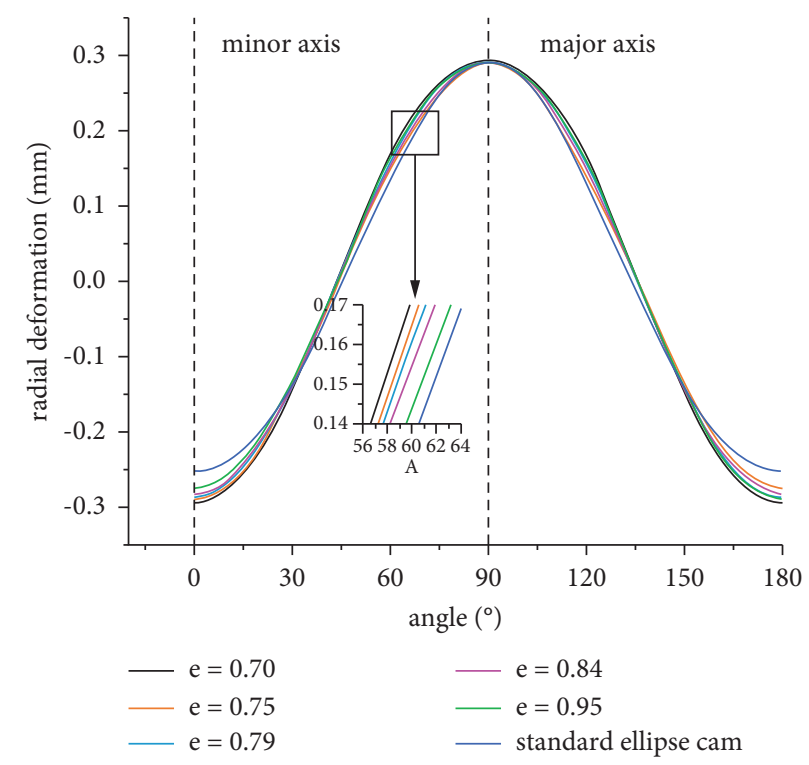

FIGURE 6: Radial deformation with different parameters.

compound curve cam with arc and spiral can effectively realize accurate deformation shape around the major axis by controlling the deformation shape of the flex-gear on the whole circumference.

From Figure 7, compared with acted by the conventional ellipse cam, the equivalent stress of the flex-gear around the major axis under the influence of the compound curve cam in different parameters showed reduction ranging from about $170 \mathrm{MPa}$ to $200 \mathrm{MPa}$. Moreover, the equivalent stress of the flex-gear around the major axis decreases with the increase of the arc segment's radius. However, if the radius of the arc segment is too large, the stress concentration near the connection point will be caused and the width of the stress plateau will be shortened. This is mainly because that increasing the radius of arc can make more teeth in meshing to gain a wider stress platform and share the load, but too large radius will lead to interference, so an appropriate radius is needed in contour design of the compound curve type. In other words, the meshing quality of the flex-gear can be easy. After parameters optimization, the equivalent stress of the 


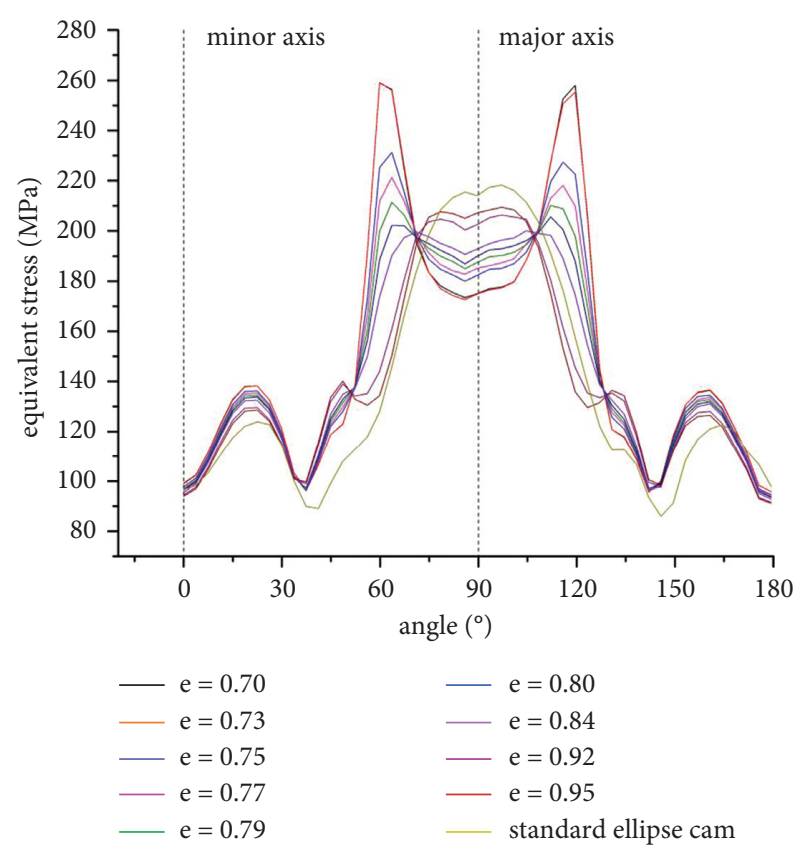

Figure 7: Equivalent stress with different parameters.

flex-gear around the major axis acted by the compound curve cam wave generator with $e=0.84, r=20.418$ can be reduced $9.92 \%$ from $214.08 \mathrm{MPa}$ to $192.84 \mathrm{MPa}$, the range of stress variation is also reduced $18.91 \%$ from $132.317 \mathrm{MPa}$ to 107.295 $\mathrm{MPa}$, and the width of the stress plateau around the major axis is increased about from $29.89^{\circ}$ to $48.58^{\circ}$.

\section{Conclusion}

In this study, the main factors affecting the accuracy and stress of the flex-gear are analyzed and discussed, including the type of stress wave generator, the type of the cam contour, and its parameters. On this basis, in order to obtain higher meshing accuracy and lower deformation stress, a compound curve cam wave generator is proposed with arc and spiral. The design method of this compound curve contour is established, numerical analysis is also carried out for its influence on the deformation of flex-gear, and parameter optimization is further carried out. The results show the following.

(1) The flex-gear can gain more accurate deformation shape under the action of the cam wave generator by shape control on the whole circumference, which is beneficial for the meshing accurate of the harmonic drive. The value and the width of deformation stress plateau around the major axis of flex-gear depend on the theoretical deformation value and the contour's corresponding curve of the wave generator.

(2) The compound curve cam with appropriate design not only can achieve better mechanical property around the meshing area but also can gain accurate deformation shape of the flex-gear. The double eccentric arc wave generator can indeed reduce the deformation stress in the meshing area, but the control of the flex-gear's deformation shape is poor. Therefore, it is an effective method to improve the double eccentric arc wave generator with a compound curve.

(3) Based on the achievable flexibility of spiral with the smooth curvature change rate, a method of compound curve cam with spiral and arc is carried out. Compared with acted by the conditional ellipse cam, first of all, the deformation shape of the flex-gear around the major axis acted by the compound curve wave generator with $e=0.84, r=20.418$ is controlled very well. Second, the equivalent stress around major axis reduces $9.92 \%$, the width of the stress platform around the major axis increases about from $29.89^{\circ}$ to $48.58^{\circ}$, and the range of stress variation also reduces $18.91 \%$.

\section{Data Availability}

The data that support the findings of this study are available from the corresponding author upon request.

\section{Conflicts of Interest}

The authors declare that they have no conflicts of interest.

\section{References}

[1] Y. Shen and Q. Ye, Theory and Design of Harmonic Gear Transmission, China Machine Press, Beijing, China, 1985.

[2] Y. He, P. Zou, Z. Zhu et al., "Design and application of a flexure-based oscillation mechanism for surface texturing," Journal of Manufacturing Processes, vol. 32, pp. 298-306, 2018.

[3] D. Meng, Z. Hu, P. Wu, S.-P. Zhu, J. A. Correia, and A. M. P. De Jesus, "Reliability-based optimisation for offshore structures using saddlepoint approximation," Proceedings of the Institution of Civil Engineers - Maritime Engineering, vol. 173, no. 2, pp. 33-42, 2020.

[4] D. Meng, T. Xie, P. Wu, S.-P. Zhu, Z. Hu, and Y. Li, "Uncertainty-based design and optimization using first order saddle point Approximation method for multidisciplinary engineering systems," ASCE-ASME Journal of Risk and Uncertainty in Engineering Systems, Part A: Civil Engineering, vol. 6, no. 3, Article ID 04020028, 2020.

[5] P. Zhi, Y. Li, B. Chen, M. Li, and G. Liu, "Fuzzy optimization design-based multi-level response surface of bogie frame," International Journal of Structural Integrity, vol. 10, no. 2, pp. 134-148, 2019.

[6] Y.-H. Li, Z. Sheng, P. Zhi, and D. Li, "Multi-objective optimization design of anti-rolling torsion bar based on modified NSGA-III algorithm," International Journal of Structural Integrity, vol. 12, no. 1, pp. 17-30, 2019.

[7] Y.-S. Eloy, I. Gonzalez-Perez, and F.-A. Alfonso, "Stress analysis of strain wave gear drives with four different geometries of wave generator," Meccanica, vol. 55, no. 11, pp. 2285-2304, 2020.

[8] G. Federico, Mucino, V. Hugo, and P. Ettore, "Influence of wave generator profile on the pure kinematic error and centrodes of harmonic drive," Mechanism and Machine Theory, vol. 104, pp. 100-117, 2016.

[9] Bhabani, S. Mahanto, V. Sahoo, and R. Maiti, "Effect of cam insertion on stresses in harmonic drive in industrial robotic 
joints," Procedia Computer Science, vol. 133, pp. 432-439, 2018.

[10] L. Li, Static and dynamic analysis of flexspline and flexible bearing under the cam with typical profile, Ph.D. Thesis, Harbin Institute of Technology, Harbin, China, 2016.

[11] B. Routh, R. Maiti, and A. K. Ray, "Aspects of lubrication at the wave generator - flexspline interface in strain wave gearing units," International Gear Conference, vol. 8, pp. 26-28, 2014.

[12] V. Kosse, "Analytical investigation of the change in phase angle between the wave generator and the teeth meshing zone in high-torque mechanical harmonic drives," Mechanism and Machine Theory, vol. 32, no. 5, pp. 533-538, 1997.

[13] Y. Fan and H. Wang, "Study on the deformation of flexspline in the engagement output harmonic drive," Journal of Nanjing University of Science and Technology, vol. 20, no. 1, pp. 38-42, 1996.

[14] S. Han and S. Hoon, "A study on stress and vibration analysis of a steel and hybrid flexspline for harmonic drive," Composite Structures, vol. 48, no. 1, pp. 827-833, 1999.

[15] S. Hu, T. Yao, H. Qin, Y. Zhang, and B. Yang, "Research of internal stress distribution of double arc flexspline," Journal of Mechanical Transmission, vol. 44, no. 05, pp. 23-28, 2020.

[16] Y. Pei, Harmonic Gear Driving Device and the Short Flexspline study[D], China Academy of Machinery Science and Technology, Beijing, 2006.

[17] W. Ostapski, "Analysis of the stress state in the harmonic drive generator-flexspline system in relation to selected structural parameters and manufacturing deviations," Bulletin of the Polish Academy of Sciences, Technical Sciences, vol. 58, no. 4, pp. 683-698, 2010.

[18] W. Ostapski and I. Mukha, "Stress state analysis of harmonic drive elements by FEM," Bulletin of the Polish Academy of Sciences, vol. 55, no. 1, pp. 115-123, 2007.

[19] V. Sahoo and M. Rathindranath, "Load sharing by tooth pairs in involute toothed harmonic drive with conventional wave generator cam," Meccanica, vol. 53, no. 1, pp. 33-394, 2018.

[20] Y. Yang, J. Wang, Q. Zhou, J. Zhu, and W. Yang, "Exact solution for conjugate profiles of zero backlash harmonic drives with elliptical cam wave generators," Journal of Central South University, vol. 48, no. 12, pp. 3231-3238, 2017.

[21] J. Xing, Y. Wang, and X. Chen, "Research on deformation and stress of flexspline in harmonic drive with cam wave generator. Machine Design and Research," in Proceedings of The 10th International Conference on Chinese Institutions and Machine Science Applications (2013CCAMMS), pp. 147-150, Shanxi, China, December 2013.

[22] H. B. Xin, H. N. Mo, J. C. Gao et al., "Study on the gear tooth influence coefficients of flexspline of harmonic drive," Advanced Materials Research, vol. 774-776, pp. 144-147, 2013.

[23] L. Zhang, X. Liu, C. Wang, W. Leyu, X. Qiao, and R. Wang, "Influence of radial deformation on stress of flexspline and meshing characteristic of harmonic reducer," Journal of Mechanical Transmission, vol. 41, no. 9, pp. 166-169, 2017.

[24] H. Dong and D. Wang, "Elastic deformation characteristic of the flexspline in harmonic drive," in Proceedings of the ASME/ IFTOMM, International Conference on Reconfigurable Mechanisms and Robots, pp. 363-369, London, UK, July 2009. 